\title{
Liquid-State 13C Polarization of $30 \%$ through Photoinduced Nonpersistent Radicals
}

Capozzi, Andrea; Karlsson, Magnus; Petersen, Jan Raagaard; Lerche, Mathilde Hauge; ArdenkjærLarsen, Jan Henrik

Published in:

Journal of Physical Chemistry C

Link to article, DOI:

10.1021/acs.jpcc.8b01482

Publication date:

2018

Document Version

Peer reviewed version

Link back to DTU Orbit

Citation (APA):

Capozzi, A., Karlsson, M., Petersen, J. R., Lerche, M. H., \& Ardenkjær-Larsen, J. H. (2018). Liquid-State ${ }^{13} \mathrm{C}$ Polarization of $30 \%$ through Photoinduced Nonpersistent Radicals. Journal of Physical Chemistry C, 122(13), 7432-7443. https://doi.org/10.1021/acs.jpcc.8b01482

\section{General rights}

Copyright and moral rights for the publications made accessible in the public portal are retained by the authors and/or other copyright owners and it is a condition of accessing publications that users recognise and abide by the legal requirements associated with these rights.

- Users may download and print one copy of any publication from the public portal for the purpose of private study or research.

- You may not further distribute the material or use it for any profit-making activity or commercial gain

- You may freely distribute the URL identifying the publication in the public portal 


\section{Liquid-State ${ }^{13} \mathrm{C}$ Polarization of $30 \%$ through Photo- Induced Non-Persistent Radicals}

Andrea Capozzi, ${ }^{*}$, Magnus Karlsson, ${ }^{\S}$ Jan Raagaard Petersen, ${ }^{\S}$ Mathilde Hauge Lerche, ${ }^{\S}$ Jan

Henrik Ardenkjaer-Larsen ${ }^{\S}$.

$\S^{\S}$ Technical University of Denmark, Department of Electrical Engineering, Center for

Hyperpolarization in Magnetic Resonance, Building 349, DK-2800 Kgs Lyngby, Denmark.

\section{AUTHOR INFORMATION}

\section{Corresponding Author}

*Andrea Capozzi, e-mail: andcapo@elektro.dtu.dk. 


\section{ABSTRACT}

Hyperpolarization via dissolution Dynamic Nuclear Polarization (dDNP) is crucial to significantly increase the magnetic resonance imaging (MRI) sensitivity, opening up for in vivo real-time MRI using in particular ${ }^{13} \mathrm{C}$-labelled substrates.

The range of applications is however limited by the relatively fast decay of the nuclear spin polarization together with the constraint of having to polarize the spins near the MRI magnet. As recently demonstrated, the employment of UV-induced non-persistent radicals represents an elegant solution to tackle these drawbacks. Nevertheless, since its introduction, the spread of the technique has been prevented by the relatively low achievable polarization, slow buildup time and time-consuming sample preparation. In the present work, thanks to a thorough investigation of the radical generation step, we provide a robust protocol to enhance the efficiency and performance of the UV-radical technique. Under optimal conditions, it was possible to produce up to $60 \mathrm{mM}$ radical in less than 5 min, and reach maximum DNP enhancement with a buildup time constant of approx. $25 \mathrm{~min}$, at $6.7 \mathrm{~T}$ and $1 \mathrm{~K}$, resulting in $30 \%{ }^{13} \mathrm{C}$ liquid-state polarization. 


\section{INTRODUCTION}

In the last fifteen years, the overwhelming liquid-state nuclear polarization enhancement achievable via dissolution Dynamic Nuclear Polarization (dDNP) has proven to break ground for many applications ranging from online enzyme and polymer kinetics, to sensitive drug discovery from hyperpolarized (HP) ligand analysis and studies of real-time cell metabolism. ${ }^{1-6}$ Without doubt, the most important success is the unique opportunity to perform staging of cancer and early treatment monitoring through HP Magnetic Resonance Imaging (MRI). ${ }^{7-13}$

The DNP technique relies on a concept known since the early '50s through the pioneering works of Overhauser, Carver and Slichter: the nuclear spin polarization can be enhanced by taking advantage of the large magnetic moment associated to unpaired electron spins (i.e. radicals) placed in close vicinity. ${ }^{14-15}$ Indeed, at the same experimental conditions (i.e. magnetic field and temperature) the thermal equilibrium polarization of an electron spins system is higher than the one of a nuclear spins system by a factor equal to the ratio of the respective magnetic moments. By coupling the two systems through microwave irradiation at the appropriate frequency, the spin order can be transferred from the first to the second. The process turned out to be extremely effective at low temperature $(1-4 \mathrm{~K})$ and high magnetic field (3.5 - $7 \mathrm{~T}$ ) on dielectric amorphous solids. ${ }^{16}$

Within this experimental scenario the so-called “dissolution step” was introduced. By means of a pressurized super-heated solvent, a quick dissolution and ejection of the HP frozen solid sample from the polarizer allowed preserving the nuclear spin polarization in the liquid state. ${ }^{17-18}$ This revolutionary method presents however some serious challenges. The nuclear $\mathrm{T}_{1}$ is relatively short (i.e. seconds to a few minutes for ${ }^{13} \mathrm{C}$ ) in solution, resulting in a fast decay of the enhanced signal. As a result, polarizers currently need to be placed in close proximity to the scanner (or high- 
resolution NMR magnet) where the imaging is performed. The most effective electron paramagnetic agent for polarizing ${ }^{13} \mathrm{C}$ nuclei with DNP is the family of highly persistent trityl radicals. ${ }^{19-22}$ For the clinical use of HP substrates, the radicals should preferably be removed from the HP solution. ${ }^{10,23-24}$ Furthermore, the radical, although beneficial for the DNP process, reduces the nuclear $\mathrm{T}_{1}$, after the dissolution step.

The more recent introduction of UV-induced non-persistent radicals for dDNP, thanks to their natural decay by temperature increase, represents an alternative to circumvent the abovementioned shortcomings. ${ }^{25-27}$

The generation of labile paramagnetic species from UV-light illumination of $\alpha$-keto acids has been explained by photo-excitation of the $n-\pi^{*}$ transition (300 - $350 \mathrm{~nm}$ ) of the $\alpha$-carbonyl group, followed by efficient intersystem crossing (ISC) to an excited triplet state with a yield close to unity. ${ }^{28-30}$ This latter molecular state, is with its longer lifetime ( $\mathrm{ms}$ to s), ${ }^{31}$ chemically reactive and liable to form radicals. These paramagnetic species, are extremely unstable at room temperature, but can be "trapped" at liquid nitrogen temperature. ${ }^{32}$ The precise photochemistry behind the formation of the radicals is still not completely clear and strongly depends on the environment of the photo-excited molecules (e.g. purity and solvent). Hydrogen abstraction and/or proton coupled electron transfer have been described as possible mechanisms; in both cases, either a second $\alpha$ keto acid molecule or a solvent molecule can be considered as a partner of the radical reaction. ${ }^{30}$, 32-35

The most promising compound for HP metabolic imaging, ${ }^{13} \mathrm{C}$-labelled pyruvate, is also the compound with the best dDNP results using UV-induced non-persistent radicals. ${ }^{26-27}$ Using pyruvic acid (PA) as a precursor, UV-PA radicals have been generated and employed to successfully polarize pyruvate itself as well as other molecules, leaving after dissolution a HP 
liquid free of radicals, containing only endogenous compounds as side products (i.e. acetate and $\mathrm{CO}_{2}$ ). Moreover, these radicals have the special property to annihilate at a temperature where the DNP sample is still frozen. This crucial feature, allowing the extraction of the sample from the polarizer when it is still in the solid state, has proven to pave a way to hyperpolarization storage and transport far away from the production site. ${ }^{36-37}$

The use of UV-induced non-persistent radicals clearly holds potential to broaden the scope of dDNP. However, a ${ }^{13} \mathrm{C}$ polarization of only $10 \%$ was obtained in about $3 \mathrm{~h}$ at $7 \mathrm{~T}$ and $1 \mathrm{~K} .{ }^{26,36}$ In particular the photo-generation process of the paramagnetic species is slow (1 $\mathrm{h}$ of irradiation time) and the yield too low (about $20 \mathrm{mM}$ ) for efficient DNP especially at high magnetic field.

We have studied in detail and optimized the polarization of $\left[1-{ }^{13} \mathrm{C}\right] \mathrm{PA}$ from photo-induced nonpersistent radicals using the labeled substrate itself as precursor. Critical parameters for fast and efficient radical production have been thoroughly investigated. This includes yield and distribution of the paramagnetic centers in the DNP samples as a function of the irradiation power, irradiation time and precursor concentration. Finally, samples have been characterized by ESR and DNP at same experimental conditions.

\section{EXPERIMENTAL METHODS}

\section{a. ESR and DNP sample preparation}

All chemicals were purchased from Sigma-Aldrich, 2605 Brøndby, Denmark.

ESR samples. Neat PA or diluted (10\% to $90 \%$ of the final volume) in water or glycerol:water 60:40 (GW64) was transferred in an Eppendorf vial, mixed and sonicated at $40{ }^{\circ} \mathrm{C}$ for 5 min to efficiently degas the liquid samples improving the glass quality after freezing. Immediately afterwards a volume in the range $2.0 \pm 0.2 \mu \mathrm{L}-10.0 \pm 0.2 \mu \mathrm{L}$ was taken from the vial by means of a 
micro-pipette (1 - $20 \mu \mathrm{L})$ and added as a drop to liquid nitrogen to form one frozen bead. The diameter of the bead was measured in liquid nitrogen by means of a precooled caliber tool. The frozen bead was then transferred inside a synthetic quartz dewar (Miniscope MS 5000 ESR spectrometer compatible, Magnettech, Berlin, Germany) filled with liquid nitrogen for UVirradiation.

DNP samples. The same procedure described above was repeated for neat, 25\%, 50\% and 75\% [1$\left.{ }^{13} \mathrm{C}\right] \mathrm{PA}$ in GW64 as well as for the reference sample $\left[1-{ }^{13} \mathrm{C}\right] \mathrm{PA}: \mathrm{H}_{2} \mathrm{O} 50: 50(\mathrm{v} / \mathrm{v}) .{ }^{36}$ This time ten 4.0 $\pm 0.2 \mu \mathrm{L}$ beads were made and transferred to the quartz dewar, for a total sample volume of approx. $40 \mu \mathrm{L}$.

\section{b. ESR and DNP sample irradiation}

All ESR and DNP experiments were performed on samples irradiated using the setup reported in Figure 1. 


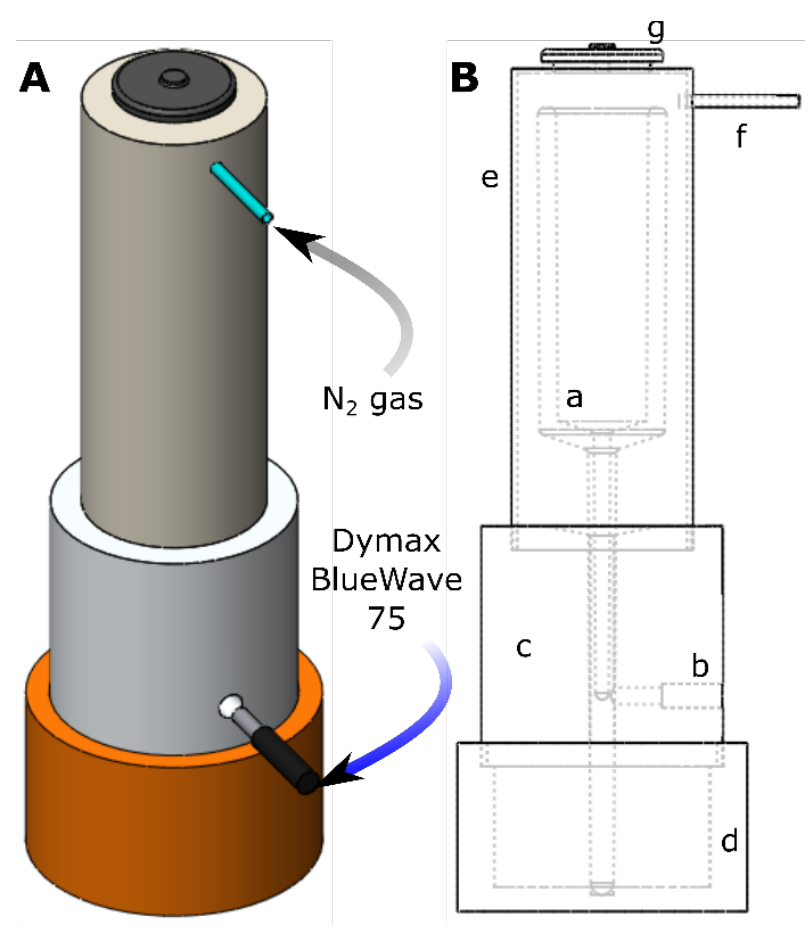

Figure 1A 3D drawing of the irradiation setup used to produce UV-radicals in the samples. B Sagittal section of the irradiation setup, all different components are visible: Miniscope MS 5000 nitrogen quartz dewar (a); Dymax BlueWave 755 mm light guide head (b); aluminum irradiation chamber (c); height adjustment 3D printed plastic foot (d); PVC cylindrical lid (e); nitrogen gas pipe (f); overpressure valve (g).

The leading idea for the construction of the "irradiation stand" was to provide a fast and homogenous illumination of the samples in a moisture free environment. The latter is important to avoid any unwanted scattering of the light reaching the sample. The setup is composed of 7 parts: (a) a quartz dewar (cold finger) containing the frozen beads immersed in liquid nitrogen; (b) a broad-band deuterium UV-light source Dymax BlueWave 75 (Dymax, Torrington, CT, USA) with maximum power of $19 \mathrm{~W} / \mathrm{cm}^{2}$; (c) an aluminum irradiation chamber that reflects the UV-light all around the sample and dissipates the heat delivered by the source; (d) an exchangeable 3D printed plastic foot to adapt the setup to quartz dewars with different tail lengths; (e) a tight-fit PVC plastic lid to keep the liquid nitrogen's surface isolated from the external environment; (f) a $5 \mathrm{~mm}$ plastic 
pipe connected to a nitrogen gas bottle to avoid any moisture contamination inside the quartz dewar; (g) an overpressure valve to compensate for liquid nitrogen bursts due to the irradiation heating.

A homogenous illumination of the sample's surface is favored by the reflecting aluminum chamber together with the tumbling of the beads from the nitrogen boiling during the irradiation. Thus, it is not necessary to stop the illumination and rotate the quartz dewar to improve an even distribution of the UV-light on the sample, as previously reported. ${ }^{26-27,} 36$ All samples for ESR experiments were irradiated in successive time steps. After each irradiation step the quartz dewar was inserted in the ESR spectrometer for measurements. If not specified the UV-source was operated at full power (refer to Supporting Information, Figure S1 for the UV-light source power calibration).

For DNP samples, the ten frozen beads were irradiated together for a time interval corresponding to approx. the maximum radical concentration.

In order to compare the performance of the irradiation setup described above with literature, one 4.0 $\pm 0.2 \mu \mathrm{L}$ frozen bead of PA: $\mathrm{H}_{2} \mathrm{O} 50: 50(\mathrm{v} / \mathrm{v})$ was irradiated outside from the stand reported in Figure 1 using the $1.3 \mathrm{~W} / \mathrm{cm}^{2} 365 \mathrm{~nm}$ LED UV source Hamamatsu LC-L5 L11403-1104 (Hamamatsu Photonics KK, Hamamatsu City, Shizuoka, Japan) employed in previous studies. ${ }^{26-}$ 27,36

\section{c. X-band ESR measurements at liquid nitrogen temperature}

For all experiments the X-band ESR spectrometer MiniScope 5000 (Magnettech, Berlin, Germany) was used. The spectrometer parameters, kept constant for all measurements, were optimized to avoid any saturation or line broadening of the ESR signal, i.e. center of the sweep = $338 \mathrm{mT}$; sweep range $=20 \mathrm{mT}$; sweep time $=20 \mathrm{~s}$; modulation frequency $=100 \mathrm{kHz}$; modulation 
amplitude $=0.1 \mathrm{mT}$; microwave power $=0.2 \mathrm{~mW}$. The radical concentration was calculated from the spectrum double integral by means of a calibration curve obtained from a series of $4.0 \pm 0.2 \mu \mathrm{L}$ frozen beads of GW64 with known concentrations of 4-hydroxy-TEMPO (12.5 - $100 \mathrm{mM}$ ) (see Supporting Information, Figure S2). All measurements were repeated twice. Data were processed in MATLAB (Mathworks, Natick, MA, USA) and OriginPro (OriginLab Corporation, Northampton, MA, USA).

\section{d. X-band ESR investigation of the radical stability}

The MiniScope 5000 was equipped with a variable temperature unit and the resonant cavity temperature set to $95 \mathrm{~K}$ (instrument minimum temperature). A frozen bead of $4.0 \pm 0.2 \mu \mathrm{L}$ neat PA and PA diluted in equal volume with water or GW64 was prepared as previously described and irradiated for $200 \mathrm{~s}$. The sample was then transferred to a precooled $5 \mathrm{~mm}$ ESR tube previously placed inside the resonant cavity. The temperature was changed in the range $95 \mathrm{~K}$ - $270 \mathrm{~K}$; before acquiring an ESR spectrum at a given temperature the variable temperature unit was allowed to stabilize for 10 s. Data were processed in MATLAB (Mathworks, Natick, MA, USA) and OriginPro (OriginLab Corporation, Northampton, MA, USA)

\section{e. Solid-state DNP}

All DNP measurements were performed on a homebuilt dDNP polarizer (6.7 $\mathrm{T}$ magnet from Magnex, Oxford, UK) working at $1.15 \pm 0.05 \mathrm{~K}$ conceptually similar to the idea introduced in 2003. ${ }^{17}$ The sample insertion unit was modified to accommodate the fluid path (FP) dissolution system. ${ }^{38}$ Microwaves were delivered from a $94 \mathrm{GHz}$ solid-state source VCOM-10/94-WPT (ELVA-1, St. Petersburg, Russia) coupled to a 200X2R4 frequency doubler (VDI, Charlottesville, VA, USA), which provided an output power of 55mW at $188 \mathrm{GHz}$. The source, digitally controlled 
through a NI-DAQ device USB-6525 (National Instruments, Austin, TX, USA) had a tuning range of $\pm 0.6 \mathrm{GHz}$ and the possibility to modulate the output frequency at a speed up to $2 \mathrm{kHz}$ and with an amplitude up to $100 \mathrm{MHz}$. A more detailed description of the system was reported previously. ${ }^{24}$, ${ }^{39}$ All ${ }^{13} \mathrm{C}$ NMR acquisitions were performed using a Varian INOVA console (Palo Alto, CA, USA) connected to a low temperature probe, placed inside the polarizer VTI (Variable Temperature Insert), remotely tuned to $71.8 \mathrm{MHz}$. The flip angle used for all acquisitions was of approx $1^{\circ}$ (i.e. pulse length $=5 \mu$ s; transmitted power $=5 \mathrm{~W}$ ). The microwave frequency giving the maximum DNP enhancement was found by sweeping the latter in steps of $20 \mathrm{MHz}$. At each microwave frequency the buildup lasted for $60 \mathrm{~min}$; afterwards the NMR signal was destroyed with a comb of $50000 \mathrm{rf}$ pulses separated by $20 \mu \mathrm{s}$, before passing to the next frequency step. The microwave modulation amplitude and frequency were investigated in the same way.

The polarization buildup was monitored by pulsing every $300 \mathrm{~s}$. Once the maximum polarization was achieved, the signal was averaged 100 times with a repetition time of $10 \mathrm{~ms}$ and the integral of the peak was calculated. After having switched off the microwaves and saturated any residual signal with the $50000 \mathrm{rf}$ pulses comb, the thermal equilibrium buildup was monitored overnight. The NMR signal was acquired every hour (100 average) until complete relaxation was achieved; the integral of the peak corresponding to thermal equilibrium was then evaluated. The DNP enhancement was calculated dividing thermal equilibrium and DNP signals. All measurements were repeated three times. It is important to stress two features: a $10 \mathrm{~ms}$ interval was enough to dephase any residual transverse magnetization; using $1^{\circ}$ pulses, the signal intensity relative to the first and the $100^{\text {th }}$ acquisition differ by $1.5 \%$.

All data were processed with MNOVA (Mestrelab Research, Santiago de Compostela, Spain) and OriginPro (OriginLab Corporation, Northampton, MA,USA). 


\section{e. UV sample handling and loading into the polarizer}

After irradiation, samples prepared for dDNP purposes were poured into a semispherical dewar filled with liquid nitrogen. Depending on the solvent used to prepare the sample, the UV-induced radicals were stable below $170 \mathrm{~K}$ at least (see Supporting Information, Figure S3). From liquid nitrogen to the latter temperature, the frozen beads could be warmed up and cooled down several times following different temperature cycles without any apparent loss of paramagnetic centers. This property provides a temperature window of about $100 \mathrm{~K}$ that guarantees the stability of the radicals during handling and loading of the samples into the polarizer. For the dDNP experiments a modification of the FP vial was required (see Figure 2).
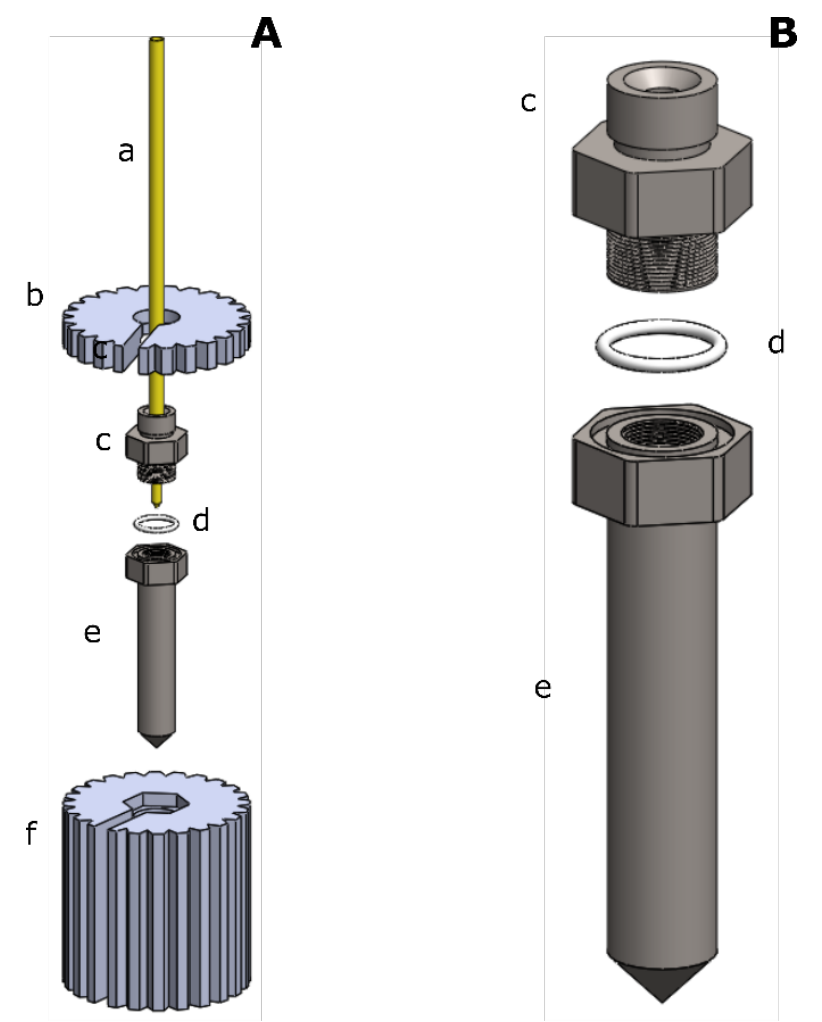

Figure 2A 3D drawing of the assembling procedure of the modified FP's bottom part. The different component are: FP's coaxial tube (a); top wrench (b); vial's neck (c); PTFE o-ring (d); vial's body (e); bottom wrench (f). B Zoom-in of the threaded vial. 
The vial is divided into two parts made of PEEK ${ }^{\circledR}$ plastic: the neck (c) and the body (e) able to contain up to $1 \mathrm{~mL}$ of sample. Only for the first use of the modified FP the vial neck was attached to the outer part of the coaxial tube (a) by means of the UV-adhesive Dymax 215-C (Dymax, Torrington, CT, USA); the latter was cured for $10 \mathrm{~s}$ using the same UV lamp employed to generate the radicals. The bottom wrench (f) immersed halfway in liquid nitrogen was used as stand for the vial body (e) during the transfer of the frozen beads through the $7 \mathrm{~mm}$ diameter top opening. The PTFE o-ring was then put in place and compressed screwing the vial neck into the body by means of the top wrench (b). The rest of the operations i.e. loading of the dissolution buffer, flushing of the FP inner volume with helium gas, leak tightness control, insertion of the FP into the polarizer were performed as previously described. ${ }^{24,39}$ On reuse the vial was opened, dried and only the PTFE o-ring was replaced with a new.

The threaded vial was also tested on a standard DNP samples of $\left[1-{ }^{13} \mathrm{C}\right] \mathrm{PA}$ doped with $30 \mathrm{mM}$ of OXO63 trityl radical. $40 \mu \mathrm{L}$ of solution were transferred into the vial, the latter was sealed and then the reported procedure was followed. ${ }^{39}$ Dissolution performance and polarization compared well with a standard FP.

\section{f. Dissolution and liquid state measurements}

The FP syringe was loaded with $15 \mathrm{~mL}$ phosphate buffer and placed into the pressure/heater module. The liquid was heated to $130^{\circ}$ and left to stabilize for $30 \mathrm{~min}$. When the maximum polarization was achieved in the solid state, the vial was lifted above the liquid helium level $(\approx 10$ $\mathrm{cm}$ ), the FP three-way valve opened and the 16 bar driven piston released inside the syringe (10 s pushing time). $11.5 \pm 0.5 \mathrm{~mL}$ of $\mathrm{HP}$ solution were collected into a receiver placed close to the polarizer. The receiver contained $10 \mathrm{M} \mathrm{NaOH}$ solution adjusted to neutralize the PA. $500 \mu \mathrm{L}$ of 
HP solution were extracted from the receiver and quickly transferred into a 5 mm NMR tube. The NMR tube was then inserted into a $400 \mathrm{MHz}$ high-resolution magnet (Agilent, Palo Alto, CA, USA) for measurements. The decay of the ${ }^{13} \mathrm{C}$ HP signal was measured every $3 \mathrm{~s}$ with $5^{\circ}$ pulse. Once complete relaxation was achieved the liquid sample in the NMR tube was doped with $10 \mu \mathrm{L}$ of Dotarem ${ }^{\circledR}$ and reinserted into the spectrometer. The same $5^{\circ}$ pulse was used to measure the signal corresponding to thermal equilibrium from 400 averages with 100 ms repetition time. The DNP enhancement was obtained computing the ratio between the value of the integral of the first spectrum of the HP decay and the thermal equilibrium one $(\mathrm{n}=3)$.

\section{g. LOD-ESR measurements}

ESR spectra of UV-irradiated $\left[1-{ }^{13} \mathrm{C}\right] \mathrm{PA}: G W 64$ 1:1 (v/v) were measured under DNP conditions (i.e. $6.7 \mathrm{~T}$ and $1.10 \pm 0.05 \mathrm{~K}$ ) using a homemade setup inspired from the work of Granwehr et al. ${ }^{40}$ and adapted to the FP loading sample system. The 3D drawings of the longitudinal detection (LOD) probe are reported in Figure 3.

The bottom part of the probe had the same dimensions of a normal FP vial in order to easily slide it into the polarizer microwave cavity without any modification of the NMR coil former. The probe was composed of five parts: a PEEK ${ }^{\circledR}$ plastic sample cup (e); a PEEK ${ }^{\circledR}$ plastic coil former with a $4 \mathrm{~mm}$ gap in the middle to allow microwaves to reach the sample (at $188 \mathrm{GHz}$ the cutoff width is $\lambda / 2=0.8 \mathrm{~mm}$ ); a FP's outer tube spare glued to the coil former as previously described (a); a 400 turn split solenoid coil realized with a $0.1 \mathrm{~mm}$ copper wire (c); a twisted pair connected to the two ends of the coil and coming out from the polarizer through the FP's outer tube (not shown). Because of the intrinsic low sensitivity of the technique, $40 \mathrm{UV}$-irradiated frozen beads were prepared and transferred into the cup while keeping it in liquid nitrogen. The rest of the probe was 
then precooled and slit/locked on the cup; the assembly was then quickly introduced into the polarizer. The two ends of the coil were connected to a $80 \mathrm{~dB}$ gain differential amplifier. The output of the amplifier was sampled and processed by a digital lock-in in LabView (National Instruments, Austin, TX, USA).

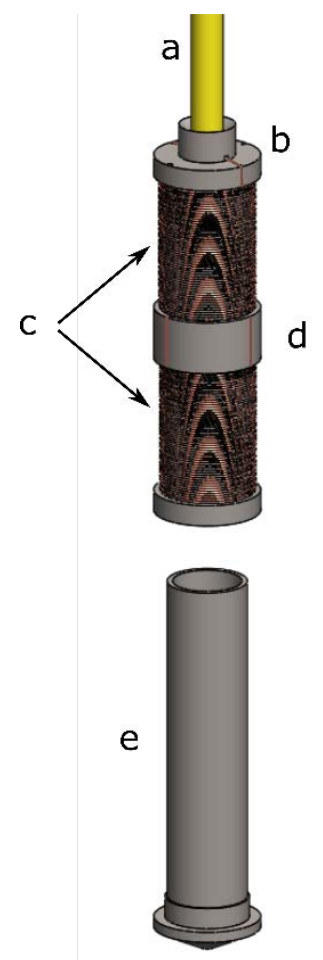

Figure 3 3D drawing of the LOD-ESR probe used to acquire the UV-irradiated $\left[1-{ }^{13} \mathrm{C}\right] \mathrm{PA}: G W 64$ 1:1 (v/v) spectrum in DNP conditions (i.e. $6.7 \mathrm{~T}$ and $1.10 \pm 0.05 \mathrm{~K}$ ). The probe is composed by a $1 \mathrm{~mL}$ PEEK ${ }^{\circledR}$ plastic sample cup (e); a PEEK ${ }^{\circledR}$ plastic coil holder (b) glued to a FP’s outer tube spare; a 400 turn split solenoid coil (c).

A schematic of the electronics involved is reported in Supporting Information, Figure S4. The ESR spectrum was obtained by sweeping the microwave frequency over the full range in steps of 5 MHz. For each frequency, the output power was modulated from $0 \mathrm{~mW}$ to $55 \mathrm{~mW}$ at a frequency of $5 \mathrm{~Hz}$. The intensity of the demodulated signal, proportional to the amount of electron spins resonating at the given frequency, was plotted as a function of the microwave frequency. A 
frequency of $5 \mathrm{~Hz}$ was found to be a good compromise between efficiency of the lock-in amplifier and intensity of the signal. Modulating at higher frequency, beneficial from the lock-in point of view, caused the saturation of the electron spins, and a reduction of the signal. On the other hand, a slower modulation $(0.5 \mathrm{~Hz})$ of the microwave power, allowed visualization of the full signal evolution across the detection coil induced by the electron spins. This procedure was used to measure the characteristic relaxation time of the electron spins at a given microwave frequency (see Supporting Information, Figure S5). It is worth noticing that the LOD-ESR pick-up coil is not resonant, so the same setup can be used at any magnetic field strength, as far as the appropriate microwave source is available.

\section{g. Low-temperature UV-vis absorption measurements}

UV-vis absorption measurements of neat PA, PA: $\mathrm{H}_{2} \mathrm{O}$ 50:50 (v/v) and PA:GW64 50:50 (v/v) at $77 \mathrm{~K}$ and $293 \mathrm{~K}$ were performed to investigate any change with temperature. A UV-vis spectrometer DH-2000 (Ocean Optics, Dunedin, FL, USA) was used, and the cuvette support placed in a polystyrene box filled with liquid nitrogen. In order to limit the amount of scattered light, a 0.1 mm light path quartz SUPRASIL cuvette (Hellma Analytics, Germany) was employed. 


\section{RESULTS AND DISCUSSION}

\section{a. Optimized irradiation setup performances}
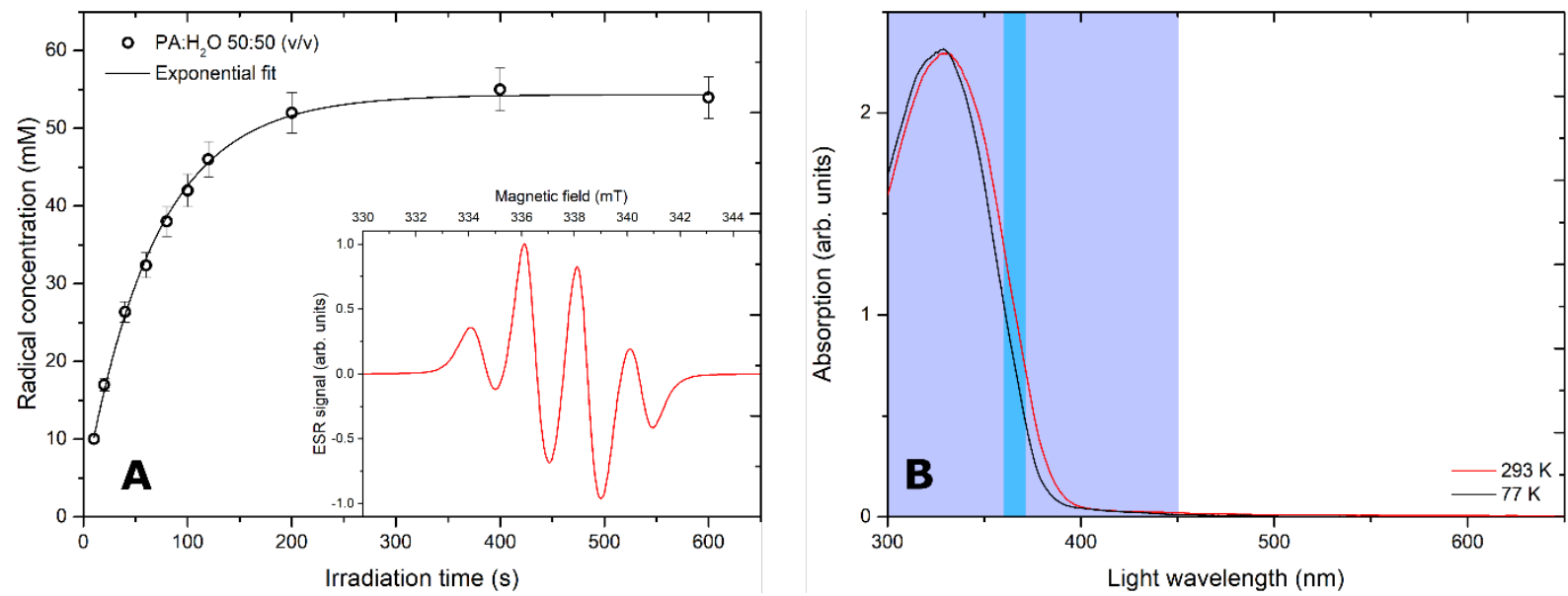

Figure 4A Radical generation time evolution measured on a 4.0 $\pm 0.2 \mu \mathrm{L}$ frozen bead of $\mathrm{PA}: \mathrm{H}_{2} \mathrm{O} 50: 50(\mathrm{v} / \mathrm{v})$; the black circles represent the experimental data points, the black line a mono-exponential fit. In the inset is shown the X-band ESR spectrum after $600 \mathrm{~s}$ of UV-irradiation. B UV-vis absorption spectrum of $\mathrm{PA}: \mathrm{H}_{2} \mathrm{O}$ 50:50 (v/v) at room temperature (red solid line) and liquid nitrogen temperature (black solid line); the area colored in violet represents the wavelength range covered by the Dymax BlueWave 75; the area in blue represents the wavelength range covered by the Hamamatsu LC-L5. Neat PA and PA diluted in GW64 showed very similar results.

In Figure 4A the radical generation time evolution measured on a $4.0 \pm 0.2 \mu \mathrm{L}$ frozen bead of PA: $\mathrm{H}_{2} \mathrm{O} 50: 50(\mathrm{v} / \mathrm{v})$ is reported. Operating the light source at the maximum power, it was sufficient to irradiate the sample for approx. $300 \mathrm{~s}$ to achieve a radical concentration $\left(C_{\infty}\right)$ of $55 \pm 3 \mathrm{mM}$. The same experiment was repeated irradiating the sample outside from the stand shown in Figure 1 and using the UV-source Hamamatsu LC-L5, employed in previous studies (see Supporting Information, Figure S6). In this case, the radical concentration was halved and the time constant twelve times longer, in agreement with literature. ${ }^{26-27,36}$ No differences were observed in the ESR spectrum, indicating that the same radical species was generated (see inset). While the increase of 
radical yield will be discussed in the next sections, the faster generation time can be understood looking at Figure 4B, where the ranges of wavelengths covered by the two light sources are superimposed to the UV-vis absorption spectrum of PA: $\mathrm{H}_{2} \mathrm{O}$ 50:50 (v/v). A higher number of photons per unit of time together with a full coverage of the PA $n-\pi^{*}$ transition explains why the BlueWave 75 UV-source dramatically shorten the radical generation process.

Saturation of the radical generation is in good agreement with Guzman et al. for a temperature close to $273 \mathrm{~K} \cdot{ }^{41}$ This behavior was assigned to the existence of a PA regeneration pathway in the photochemical reaction (null cycle), with steady state value of the radical concentration depending on the rates of the different reactions involved. Fitting the data points to the exponential relation, typical of first-order kinetic reactions,

$$
C(t)=C_{\infty}(1-\exp (-t / \tau))
$$
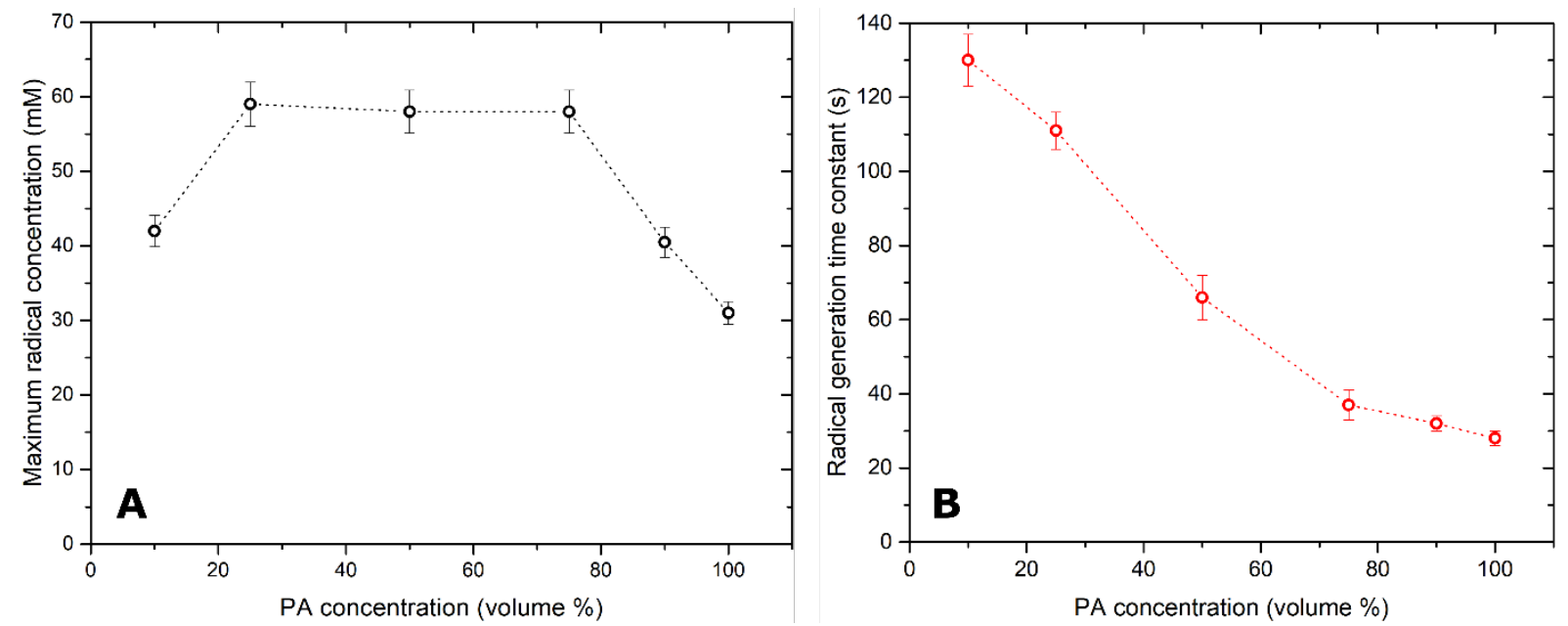

Figure 5A Maximum radical concentration $\left(C_{\infty}\right)$ as a function of the PA concentration in GW64. B For the same kind of samples the $\tau$ parameter of equation (1) is reported. All measurements were performed on a single $4.0 \pm 0.2 \mu \mathrm{L}$ frozen bead $(n=2)$. 
a radical generation time constant $\tau=67 \pm 2 \mathrm{~s}$ was estimated. Similar results were obtained by replacing the 50\% water volume with GW64; the maximum radical concentration and time constant were $C_{\infty}=58 \pm 3 \mathrm{mM}$ and $\tau=66 \pm 6 \mathrm{~s}$, respectively. Diluting the PA with this solvent mixture allowed to study the radical generation process as a function of the precursor concentration preserving the transparency and glassy property of the frozen beads. In Figure 5 and Table $1 C_{\infty}$ and $\tau$ are reported as a function of the amount of PA in the samples for $4.0 \pm 0.2 \mu \mathrm{L}$ frozen beads (for raw data see Supporting Information, Figure S7). The PA concentration was increased from $10 \%$ to $100 \%$ of the total sample volume. The radical generation time constant decreased from $130 \pm 7$ s to $28 \pm 2$ s. The maximum radical concentration showed a plateau around $58 \mathrm{mM}$ for PA concentration between $25 \%$ and $75 \%$ and lower values otherwise.
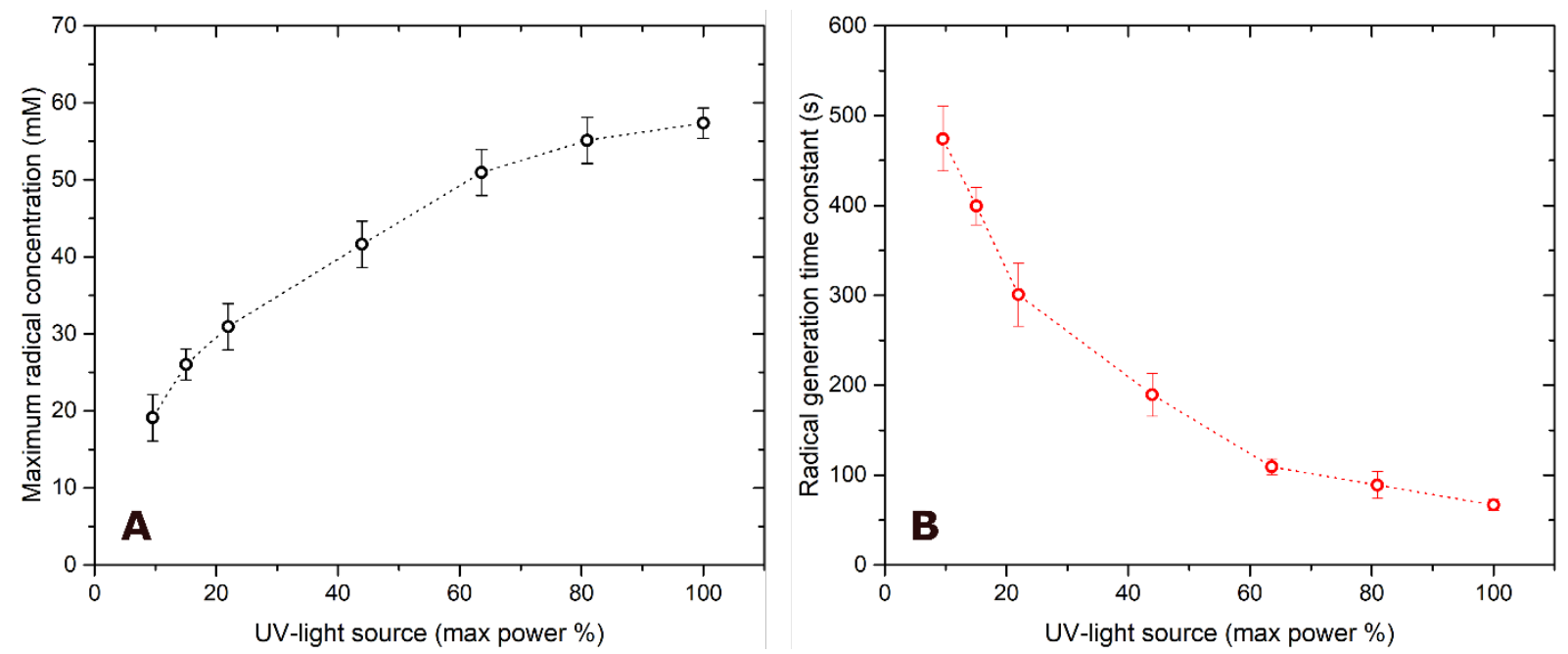

Figure 6A Maximum radical concentration $\left(C_{\infty}\right)$ as a function of the UV-source output power. B For the same power values the $\tau$ parameter of equation (1) is reported. All measurements were performed on a single $4.0 \pm 0.2 \mu \mathrm{L}$ frozen bead of a sample containing 50:50 PA:GW64 (v/v) $(\mathrm{n}=2)$.

For the sample containing PA:GW64 50:50 (v/v) a study of $C_{\infty}$ and $\tau$ as a function of the UV-light source output power was performed. The latter was changed in the range $10-100 \%$ of its value. 
Results for $4.0 \pm 0.2 \mu \mathrm{L}$ frozen beads are reported in Figure 6 and Table 2: $C_{\infty}$ and $\tau$ were respectively plotted as a function of the UV-light source output power (see Supporting Information, Figure S8 for raw data). Both the radical maximum yield and generation time constant were dependent on the power of the lamp. $C_{\infty}$ increased from $19 \pm 2 \mathrm{mM}$ to $58 \pm 3 \mathrm{mM}$ with a tendency to achieve a plateau for high powers, while $\tau$ decreased from $474 \pm 36 \mathrm{~s}$ to $66 \pm 6 \mathrm{~s}$.

A central point of the measurements showed in Figure 5 and 6 is that they were performed on spherical frozen samples of the same size. If the radical generation time constants evolve as one would expect (more precursor concentration or light power leads to faster radical generation) in both cases, the behavior of the radical maximum yield is

\begin{tabular}{|c|c|c|c|}
\hline Sample & $\begin{array}{c}\text { PA concentration } \\
\mathbf{( \% )}\end{array}$ & $\boldsymbol{C}_{\infty}(\boldsymbol{m} \boldsymbol{M})$ & $\boldsymbol{\tau}(\mathbf{s})$ \\
\hline 10:90 PA:GW64 & 10 & $42 \pm 2$ & $130 \pm 7$ \\
\hline 25:75 PA:GW64 & 25 & $59 \pm 3$ & $111 \pm 5$ \\
\hline 50:50 PA:GW64 & 50 & $58 \pm 3$ & $66 \pm 6$ \\
\hline 75:25 PA:GW64 & 75 & $58 \pm 4$ & $37 \pm 2$ \\
\hline 90:10 PA:GW64 & 90 & $40 \pm 3$ & $32 \pm 2$ \\
\hline neat PA & 100 & $31 \pm 2$ & $28 \pm 2$ \\
\hline
\end{tabular}

Table $1 C_{\infty}$ and $\tau$ arising from the fit of raw data to the expression reported in (1) are listed as a function of the PA concentration in GW64. The UV-irradiation as a function of time and X-band ESR measurements were performed on single 4.0 $\pm 0.2 \mu \mathrm{L}$ frozen beads. All measurements were repeated twice; the average and standard deviation are reported.

\begin{tabular}{|c|c|c|c|}
\hline Sample & $\begin{array}{c}\text { UV-light power } \\
\mathbf{( \% )}\end{array}$ & $\boldsymbol{C}_{\infty}(\mathbf{m} \boldsymbol{M})$ & $\boldsymbol{\tau}$ (s) \\
\hline 50:50 PA:GW64 & 10 & $19 \pm 2$ & $474 \pm 36$ \\
\hline 50:50 PA:GW64 & 15 & $26 \pm 3$ & $399 \pm 21$ \\
\hline 50:50 PA:GW64 & 22 & $31 \pm 3$ & $300 \pm 28$ \\
\hline 50:50 PA:GW64 & 44 & $42 \pm 4$ & $189 \pm 14$ \\
\hline
\end{tabular}




\begin{tabular}{|c|c|c|c|}
\hline 50:50 PA:GW64 & 64 & $51 \pm 3$ & $109 \pm 9$ \\
\hline 50:50 PA:GW64 & 81 & $55 \pm 2$ & $89 \pm 15$ \\
\hline 50:50 PA:GW64 & 100 & $58 \pm 3$ & $66 \pm 6$ \\
\hline
\end{tabular}

Table $2 C_{\infty}$ and $\tau$ arising from the fit of raw data to the expression reported in (1) are listed as a function the UV-light source output power. The UV-irradiation as a function of time and X-band ESR measurements were performed on single 4.0 $\pm 0.2 \mu \mathrm{L}$ frozen beads of 50:50 PA:GW64 (v/v). All measurements were repeated twice; the average and standard deviation are reported.

a clear indication of the fact that radicals are not homogeneously distributed within the bead, a feature pointed out previously in a similar context. ${ }^{25}$ The reason is most likely the PA strong absorption of the UV-light in the surface layer (the GW64 solution did not show any absorption in the range of wavelengths involved; data not shown). Indeed, if it was always possible to efficiently irradiate all the sample volume, one would expect an increasing radical maximum yield for increasing precursor concentrations in Figure 5A and the same plateau regardless of the UV-source output power in Figure 6A. The $n-\pi^{*}$ transition for aqueous PA takes place in the near UV with a molar extinction coefficient $\varepsilon$ of the order of $10 \mathrm{M}^{-1} \mathrm{~cm}^{-1}$ around a wavelength of $320 \mathrm{~nm} .{ }^{29}$ Thus, for undiluted $(C=14 \mathrm{M})$ and glassy PA the light linear attenuation coefficient $\mu$ is of the order of $\varepsilon \cdot C \approx 140 \mathrm{~cm}^{-1}$ that corresponds to a light penetration length of $\mu^{-1} \approx 70 \mu \mathrm{m} \cdot{ }^{42} \mathrm{~A} 4 \mu \mathrm{L}$ spherical bead has a radius of $\approx 1 \mathrm{~mm}$; it is therefore difficult to efficiently photo-excite all the PA molecules in the sample and generate the radicals with a volumetric homogeneous distribution, especially when the precursor molecules are in high concentrations.

\section{b. Distribution profile of the photo-induced radicals}

To better understand the spatial distribution of radicals within the bead, the irradiation was repeated for different sizes $(2-10 \mu \mathrm{L})$, for all samples listed in Table 1 excepted for neat PA. It 
was not possible to use neat PA because of the poor glass quality after freezing of larger droplets. For all preparations, the maximum ESR signal $(S)$ arising from each frozen bead was plotted as a function of the radius $(r)$ of the bead.

If radicals were homogenously distributed, the signal contribution $S$ ' to $S$ of the spherical shell placed at distance $x$ from the center of the bead would be

$$
S^{\prime}(x)=S_{0}\left(4 \pi x^{2}\right)
$$

such as

$$
S(r)=\int_{-r}^{0} S^{\prime}(x) d x=S_{0}\left(\frac{4}{3} \pi r^{3}\right)
$$

where $S_{0}$ is a proportionality constant depending on the ESR spectrometer parameters and the quantum yield of the photochemical reaction (see Supporting Information, Figure S11 for a proof of the method using ordinary nitroxyl radical).

Only for the least concentrated sample it was possible to accurately fit the data to the expression (3) (see Supporting Information, Figure S9). For higher PA concentration the results were poor (see Supporting Information, Figure S10 as example).

When we generate radicals by means of photo-induction, the light is exponentially attenuated through the sample with a wavelength dependent extinction coefficient. 


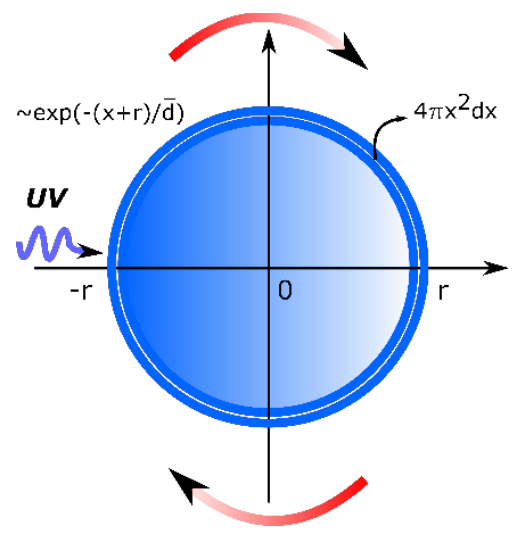

Figure 7 2D schematics of the spherical sample irradiation model. The light comes from left to right in the picture, but the problem is symmetric with respect to the center because of the random tumbling of the bead during the irradiation in liquid nitrogen (red curved arrows). The equivalent problem is to have fixed sphere with light coming under a $4 \pi$ solid angle. Thus, the photons encounter successive shells with a $4 \pi x^{2}$ surface.

As a consequence the radical precursors located at different distances from the bead surface are not evenly excited by the UV-photons. Let $\bar{\mu}$ be the mean light linear attenuation coefficient, averaged over the PA absorption peak wavelength (see Figure 4B) and $\bar{d}$ the correspondent mean penetration depth (see Figure 7 for a graphical representation of the model). In that case the signal contribution $S^{\prime}$ of the spherical shell placed at distance $x$ from the center of the bead could be tentatively modified as

$$
S^{\prime}(x)=S_{0}\left(4 \pi x^{2} \exp \left(-\frac{x+r}{\bar{d}}\right)\right)
$$

leading to the total ESR signal expression

$$
S=\int_{-r}^{0} S^{\prime}(x) d x=S_{0}\left(4 \pi\left(-2 \bar{d}^{3} \exp (-r / \bar{d})+\bar{d} r^{2}-2 \bar{d}^{2} r+2 \bar{d}^{3}\right)\right)
$$



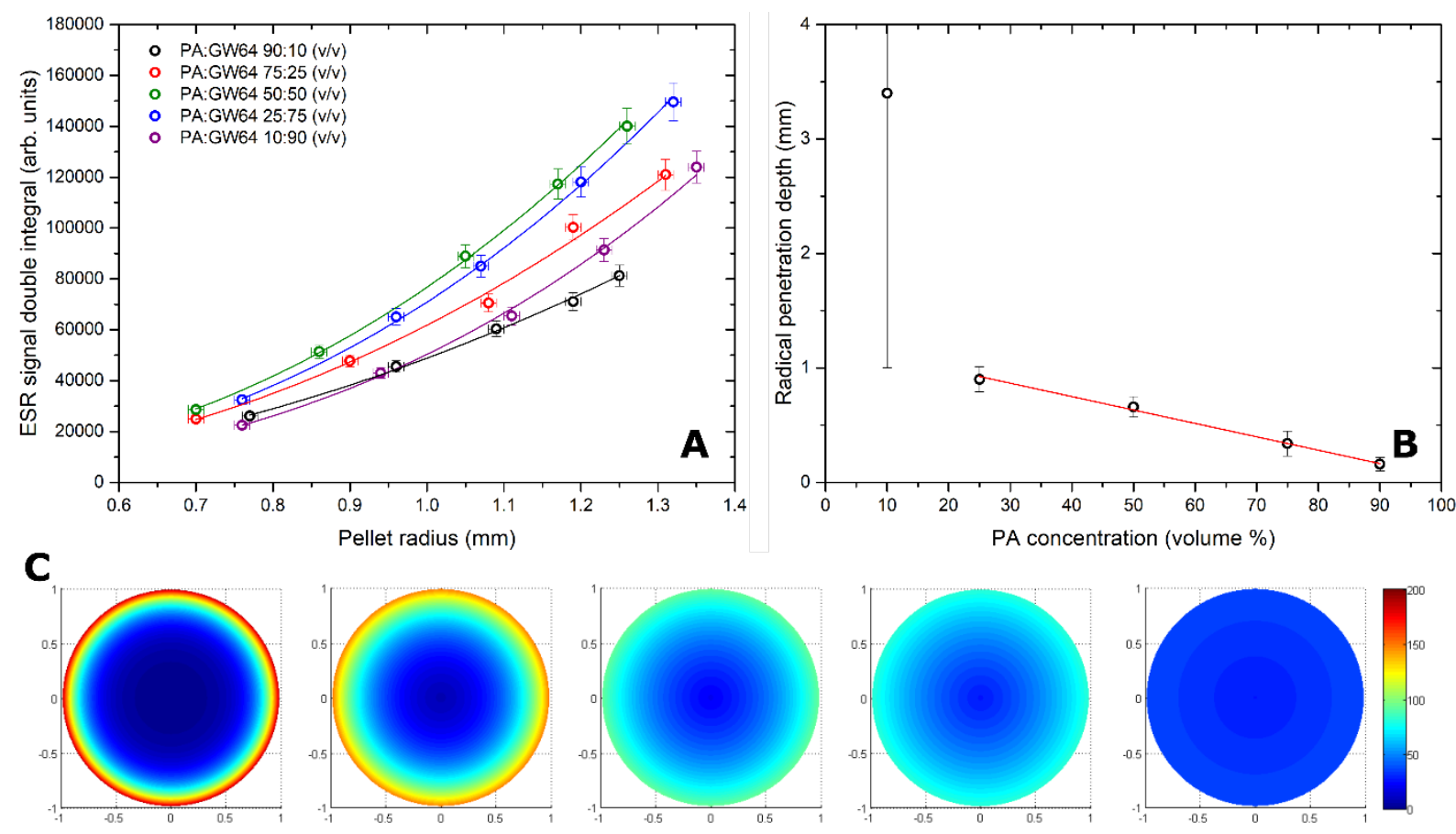

Figure 8A The value of the maximum ESR signal (circles) arising from UV-irradiation of frozen beads with different size $(2-10 \mu \mathrm{L})$ is plotted as a function of the bead radius. Each color corresponds to a different amount of PA in the sample preparation (see Table 1 for details); for each preparations, data were fitted (curves connecting the data points) to expression (4). B For each preparations the value of the $\bar{d}$ parameter, obtained from the fit, of the experimental data is plotted as a function of the PA concentration; the error bar is the uncertainty on the fit. C A simulation of the radical distribution profile in a $4 \mu \mathrm{L}$ bead is presented according to the different values of $\bar{d}$. From left to right the value of $\bar{d}$ is $0.16 \pm 0.06 \mathrm{~mm} ; 0.34 \pm 0.11 \mathrm{~mm} ; 0.66 \pm 0.09 \mathrm{~mm} ; 0.90 \pm 0.11 \mathrm{~mm} ; 3.40 \pm 2.40 \mathrm{~mm}$. The bead radius is $0.98 \mathrm{~mm}$ in all cases while the "overall-volume" radical concentration $\left(C_{\infty}\right)$ was taken from Table 1 . Axes are in mm; the color scale is in $\mathrm{mM}$.

Expression (5) was used to fit the data reported in Figure 8A. $S_{0}$ and $\bar{d}$ were free parameters of the fit; $\bar{d}$ was found to decrease linearly for increasing PA concentration (see Figure 8B). Only the data point corresponding to the 10:90 PA:GW64 (v/v) sample deviated from this trend. A conceivable explanation is that $\bar{d}$ can be accurately estimated as far as it is reasonably smaller than the radius of the bead. If this is not the case the model is unreliable, as shown by the size of the 
error bar and the fact that the purple data set reported in Figure 8A could be nicely fitted using expression (2) (see Supporting Information, Figure S9). In Figure 8C a simulation of the radicals distribution profile for a $4 \mu \mathrm{L}$ bead (bead with a radius of $0.98 \mathrm{~mm}$ ) is reported according to the five different values of $\bar{d}$ obtained from the fit and measured average radical concentration reported in Table 1.

For the PA:GW64 50:50 (v/v) the experiment reported in Figure 8A was repeated for an output power of the UV-source equal to $20 \%$ of the maximum. The $\bar{d}$ parameter was calculated to about half of the value obtained at full power (see Supporting Information, Figure S12).

According to the results reported in this session, we can give interpretation of the unusual behavior of the radical maximum yield reported in Figure 5A and Figure 6A.

The power dependence of the concentration plateau could be justified according to the following qualitative argument. Generating radicals is not the only possible pathway that photo-excited PA can follow. Phenomena like fluorescence and phosphorescence are highly probable. ${ }^{29,42-43}$ Thus, the majority of the photons are absorbed without creating any paramagnetic center. The photons that did not interact with any chromophore in the first molecular layer will interact with the second, the third and so on and so forth. At some point, accessing more and more internal molecular layer, the number of survival photons is so low that the radical reaction rate becomes too long to generate a measurable increase of the radical concentration. Thus, by increasing the UV-source's power we increase the chances of efficiently photo-excite a larger number of molecular layers in the frozen beads. As a consequence we promote the generation of more radicals (see Figure 6A).

At high concentration of PA, on one hand we have more chances to induce radicals in more external molecular layers, but on the other hand the light attenuation increases. So, what we see in 
Figure 5A is a balance between 2 effects: amount of available "targets" in a single molecular layer combined with the amount of molecular layers that can be efficiently reached by the UV-photons.

\section{c. dDNP results}

The optimization of the microwave irradiation parameters is shown for the sample containing [1$\left.{ }^{13} \mathrm{C}\right]$ PA:GW64 50:50 (v/v) and 55 \pm 3 mM radical concentration. Results are reported in Figure 9. The best DNP enhancement was achieved for an irradiation frequency of $188.025 \mathrm{GHz}$ with frequency modulation of the microwaves at a rate of $1 \mathrm{kHz}$ and amplitude of $35 \mathrm{MHz}$. Without any modulation the DNP spectrum was slightly narrower and the enhancement decreased by $50 \%$ (see Figure 9A). The observation that microwave modulation increases the nuclear spin polarization ${ }^{44-45}$ is an indication that the system is characterized by poor spectral diffusion at this field and temperature. ${ }^{46-47}$
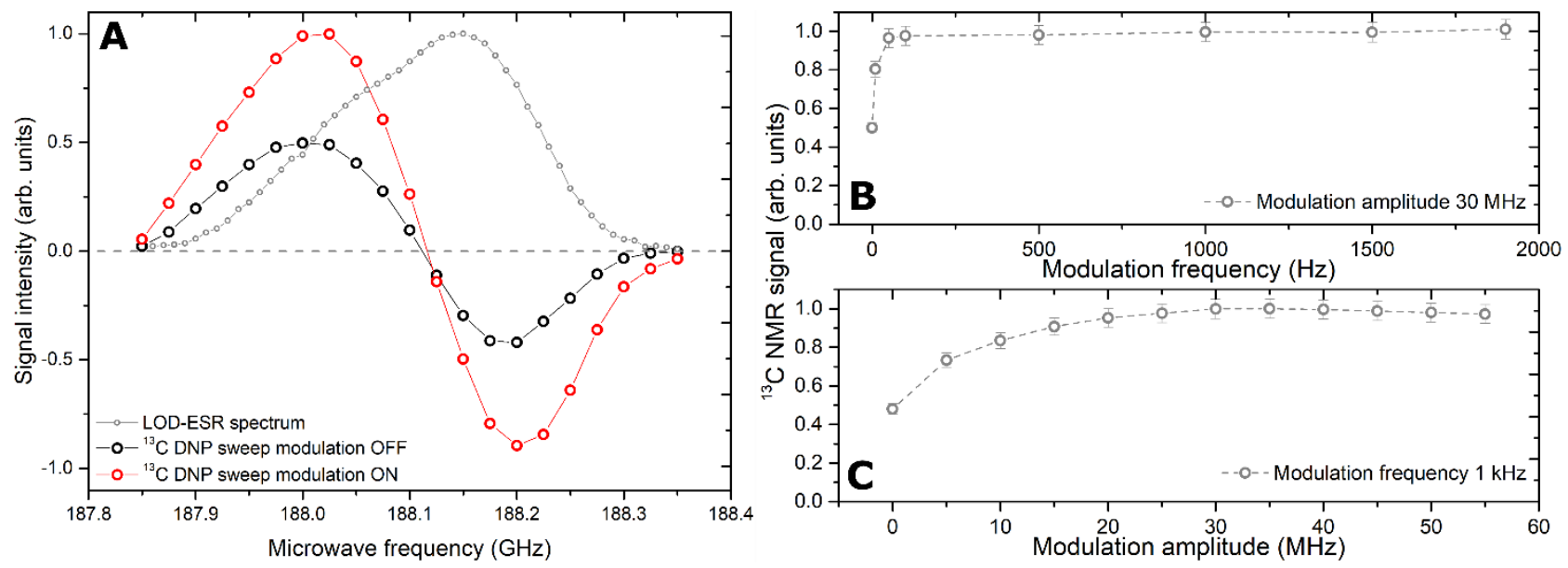

Figure 9A ${ }^{13} \mathrm{C}$ DNP spectra of PA:GW64 1:1 (v/v) irradiated $200 \mathrm{~s}$ acquired when applying $30 \mathrm{MHz}$ amplitude/1 kHz frequency microwave modulation (red circles) and without any modulation (black circles) superimposed to the sample’s LOD-ESR spectrum (grey dots) measured ad $6.7 \mathrm{~T}$ and $1.10 \pm 0.05 \mathrm{~K} . \mathbf{B}{ }^{13} \mathrm{C}$ DNP enhancement as a function of the microwave modulation frequency acquired with a main irradiation frequency of $188.025 \mathrm{GHz}$ and modulation 
amplitude of $35 \mathrm{MHz} . \mathrm{C}^{13} \mathrm{C}$ DNP enhancement as a function of the microwave modulation amplitude acquired with a main irradiation frequency of $188.025 \mathrm{GHz}$ and modulation frequency of $1 \mathrm{kHz}$.

This observation together with the overlap of the DNP and ESR spectra suggest that the mechanism involved is cross-effect rather than thermal mixing, where an extremely efficient spectral diffusion is supposed to saturate all electron spins in the system, under continuous microwave irradiation. ${ }^{48}$ Moreover by applying the microwave modulation frequency a beneficial effect was measured already for a value of $10 \mathrm{~Hz}$ and a plateau was achieved beyond $100 \mathrm{~Hz}$ (see Figure 9B). This was in good agreement with the estimation of the effective electron $T_{1}$ (combination of spin-lattice relaxation and spectral diffusion) at $188.025 \mathrm{GHz}$ of $94 \pm 2$ ms measured with the LOD-ESR probe (see Supporting Information, Figure S5). Concerning the amplitude of the microwave modulation, the ${ }^{13} \mathrm{C}$ DNP enhancement achieved a maximum for a value in the range $30-35 \mathrm{MHz}$ and then remained constant (see Figure 9C), as previously observed on ${ }^{1} \mathrm{H}$ nuclei by Bornet et al. in the case of nitroxyl radicals. ${ }^{49}$ All other DNP samples showed a very similar behavior (data not shown).

Figure 10 shows the ${ }^{13} \mathrm{C}$ polarization buildup at optimal DNP conditions $(188.025 \mathrm{GHz}$ irradiation frequency, $1 \mathrm{kHz}$ modulation frequency, $35 \mathrm{MHz}$ modulation amplitude) for neat $\left[1-{ }^{13} \mathrm{C}\right] \mathrm{PA}$ with $32 \pm 2 \mathrm{mM}$ radical (panel A), and the three PA:GW64 solutions generating the higher radical concentration (approx.. $55 \mathrm{mM}$ ) after UV-irradiation i.e. [1- $\left.{ }^{13} \mathrm{C}\right]$ PA:GW64 75:25 (v/v) (panel B), [1- $\left.{ }^{13} \mathrm{C}\right]$ PA:GW64 50:50 (v/v) (panel C) and $\left[1-{ }^{13} \mathrm{C}\right]$ PA:GW64 25:75 (v/v) (panel D). In all cases the UV-irradiation time was approx. three time constants. By fitting the data to the usual monoexponential expression

$$
P(t)=P_{\infty}\left(1-\exp \left(-t / T_{b}\right)\right)
$$


where $P(t), P_{\infty}$ and $T_{b}$ are respectively the polarization at time $t$, the polarization at equilibrium and the buildup time constant, a striking feature stand out. The two samples with higher PA concentration (panel $\mathbf{A}$ and $\mathbf{B}$ ) did not follow the (5) showing a fast buildup at the beginning of the curve then followed by a slow growth of the polarization. Moreover they reached a lower polarization level than the less concentrated samples reported in panel $\mathbf{C}$ and $\mathbf{D}$. The explanation has to be found in the radical distribution profile. As shown previously in the paper, for high PA content samples the radicals are mainly generated at the surface of the frozen beads and their concentration decreases gradually to the core following a profile that depends on the value of the $\bar{d}$ parameter. Thus, we assume that the polarization buildup under microwave irradiation can be described as the superposition of single exponential curves, each of them characterized by a time constant $\tau_{b}$ dependent on the local radical concentration. One can write: ${ }^{50}$ 

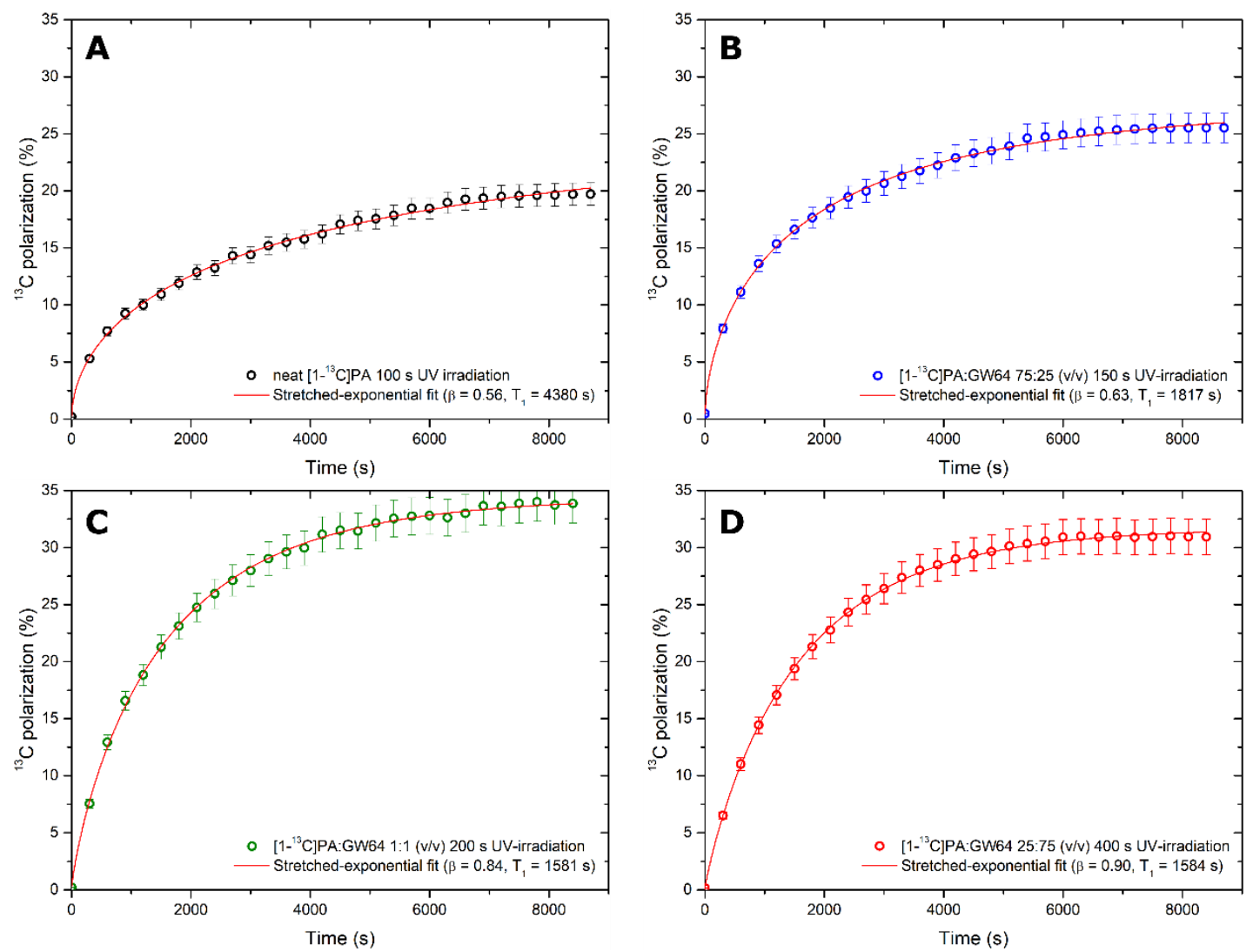

Figure $10{ }^{13} \mathrm{C}$ polarization buildup in optimal DNP conditions $(188.025 \mathrm{GHz}$ irradiation frequency, $1 \mathrm{kHz}$ modulation

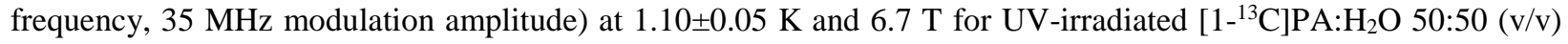
(A); neat $\left[1-{ }^{13} \mathrm{C}\right] \mathrm{PA}$ (B); $\left[1-{ }^{13} \mathrm{C}\right] \mathrm{PA}: G W 6450: 50$ (v/v) (C); [1-13 C]PA:GW64 25:75 (v/v) (D). The different samples were UV-irradiated for three time constants depending on the composition. The red curves indicate the stretchedexponential function used to fit the experimental data. The polarization values reported were measured in the solid state.

$$
P(t)=P_{\infty}\left(1-\int_{0}^{\infty} V_{13 C}\left(\tau_{b}\right) \exp \left(-t / \tau_{b}\right) d \tau_{b}\right)
$$

where $V_{13 C}\left(\tau_{b}\right)$ is the probability distribution of buildup time constants, i.e. the number of ${ }^{13} \mathrm{C}$ nuclei with polarization time constant $\tau_{b}$. If $V_{13 C}\left(\tau_{b}\right)$ decreases for larger values of $\tau_{b}$ then expression (6) gives a stretched-exponential buildup curve of the kind: 


$$
P(t)=P_{\infty}\left(1-\exp \left(-\left(t / T_{b}\right)^{\beta}\right)\right.
$$

where the value of the stretched exponent $0<\beta<1$ is determined by the gradient at which $\tau_{b}$ changes. A small value of $\beta$ means high inhomogeneity of time constants; $\beta$ very close to 1 approaches the limit of the classic mono-exponential buildup. For a more exhaustive dissertation see Kenyon et al. ${ }^{50}$ Because of the morphology of the samples investigated in the present study and the well-known feature that increasing the radical concentration decreases the DNP buildup time, ${ }^{51}$ the condition on $V_{13 C}\left(\tau_{b}\right)$ is fulfilled. Expression (7) was used to fit the data reported in Figure 10. An increase of the exponent $\beta$ was found by decreasing the PA concentration in good agreement with the radical distribution profile model introduced previously. Moreover the too high radical concentration in the more external shell of samples reported in panel $\mathbf{A}$ and $\mathbf{B}$ (see Figure 8C to have an idea of the radical distribution profile) explains why the final polarization is lower compared to what is reported in panel $\mathbf{C}$ and $\mathbf{D}$ : excessive electron local spin density leads to inefficient DNP, thus a portion of the ${ }^{13} \mathrm{C}$ nuclei will be poorly polarized decreasing the overall enhancement factor.

In order to check if the maximum achievable radical concentration was optimal for DNP at this magnetic field and temperature the sample containing $\left[1-{ }^{13} \mathrm{C}\right] \mathrm{PA}: \mathrm{GW64}$ 50:50 (v/v) was irradiated for 100 s only to reach $40 \mathrm{mM}$ radical concentration. The buildup time constant almost doubled, the polarization reduced by $20 \%$ and the $\beta$ parameter was smaller (see Supporting Information, Figure S12).

The reference sample containing $\left[1-{ }^{13} \mathrm{C}\right] \mathrm{PA}: \mathrm{H}_{2} \mathrm{O}$ 50:50 (v/v) irradiated $200 \mathrm{~s}$ was also polarized at optimal microwave irradiation conditions (see Supporting Information, Figure S13). The polarization buildup, very close to a mono-exponential curve, took longer time and the final 
polarization was lower than the sample containing the same amount of PA reported in panel $\mathbf{C}$. Probably at this field and temperature the addition of glycerol in the sample matrix improves the quality of the glass leading to more efficient DNP. ${ }^{52}$ All numerical results are reported in Table 3.

\begin{tabular}{|c|c|c|c|c|c|}
\hline Sample & $\begin{array}{c}\text { Radical } \\
\text { conc. }(\mathbf{m M})\end{array}$ & $\begin{array}{l}\text { Irradiation } \\
\text { time (s) }\end{array}$ & $\begin{array}{c}\text { Maximum }{ }^{13} \mathrm{C} \\
\text { polarization } \\
(\%)\end{array}$ & $\beta$ & $T_{b}(s)$ \\
\hline $\begin{array}{c}25: 75 \\
{[1-} \\
{ }^{13} \text { C]PA:GW64 }\end{array}$ & $54 \pm 3$ & 400 & $31.5 \pm 2.0$ & $0.90 \pm 0.03$ & $1584 \pm 50$ \\
\hline $\begin{array}{c}50: 50 \\
{[1-} \\
{ }^{13} \text { C]PA:GW64 }\end{array}$ & $55 \pm 3$ & 200 & $33.0 \pm 2.0$ & $0.84 \pm 0.02$ & $1581 \pm 45$ \\
\hline $\begin{array}{c}50: 50 \\
{[1-} \\
{ }^{13} \text { C]PA:GW64 }\end{array}$ & $40 \pm 2$ & 100 & $27.0 \pm 1.4$ & $0.78 \pm 0.02$ & $3137 \pm 100$ \\
\hline $\begin{array}{c}75: 25 \\
{[1-} \\
{ }^{13} \text { C]PA:GW64 }\end{array}$ & $56 \pm 3$ & 150 & $25.5 \pm 1.3$ & $0.63 \pm 0.01$ & $1817 \pm 65$ \\
\hline $\begin{array}{c}\text { neat } \\
{\left[1{ }^{13} \mathrm{C}\right] \mathrm{PA}}\end{array}$ & $32 \pm 2$ & 100 & $20.5 \pm 1.1$ & $0.56 \pm 0.01$ & $4380 \pm 150$ \\
\hline $\begin{array}{c}50: 50 \\
{\left[1-{ }^{13} \mathrm{C}\right] \mathrm{PA}: \mathrm{H}_{2} \mathrm{O}}\end{array}$ & $50 \pm 3$ & 200 & $27.2 \pm 1.4$ & $0.95 \pm 0.02$ & $2076 \pm 75$ \\
\hline
\end{tabular}

Table 3 Radical concentration, corresponding UV-irradiation time, maximum solid state ${ }^{13} \mathrm{C}$ polarization, buildup time and $\beta$ exponent are reported for all DNP samples polarized at $6.7 \mathrm{~T}, 1.10 \pm 0.05 \mathrm{~K}$ and $188.025 \mathrm{GHz}$ (frequency

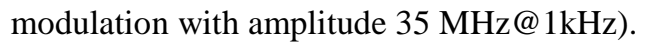

Figure 11A shows the dissolution, collection and injection of the HP sample into the $400 \mathrm{MHz}$ NMR spectrometer by means of the manual transfer system used in this study. It took about $10 \mathrm{~s}$ and caused a relative polarization loss of $5-10 \%$. The HP signal decay for the $\left[1-{ }^{13} \mathrm{C}\right] \mathrm{PA}$ :GW64 50:50 (v/v) sample is reported in Figure 11B. Data were fitted with a mono-exponential curve 
and, after correcting for the effect of the rf pulses, the liquid state $T_{1}$ was $61 \pm 1 \mathrm{~s}$, confirming the absence of radicals after dissolution. ${ }^{27}$

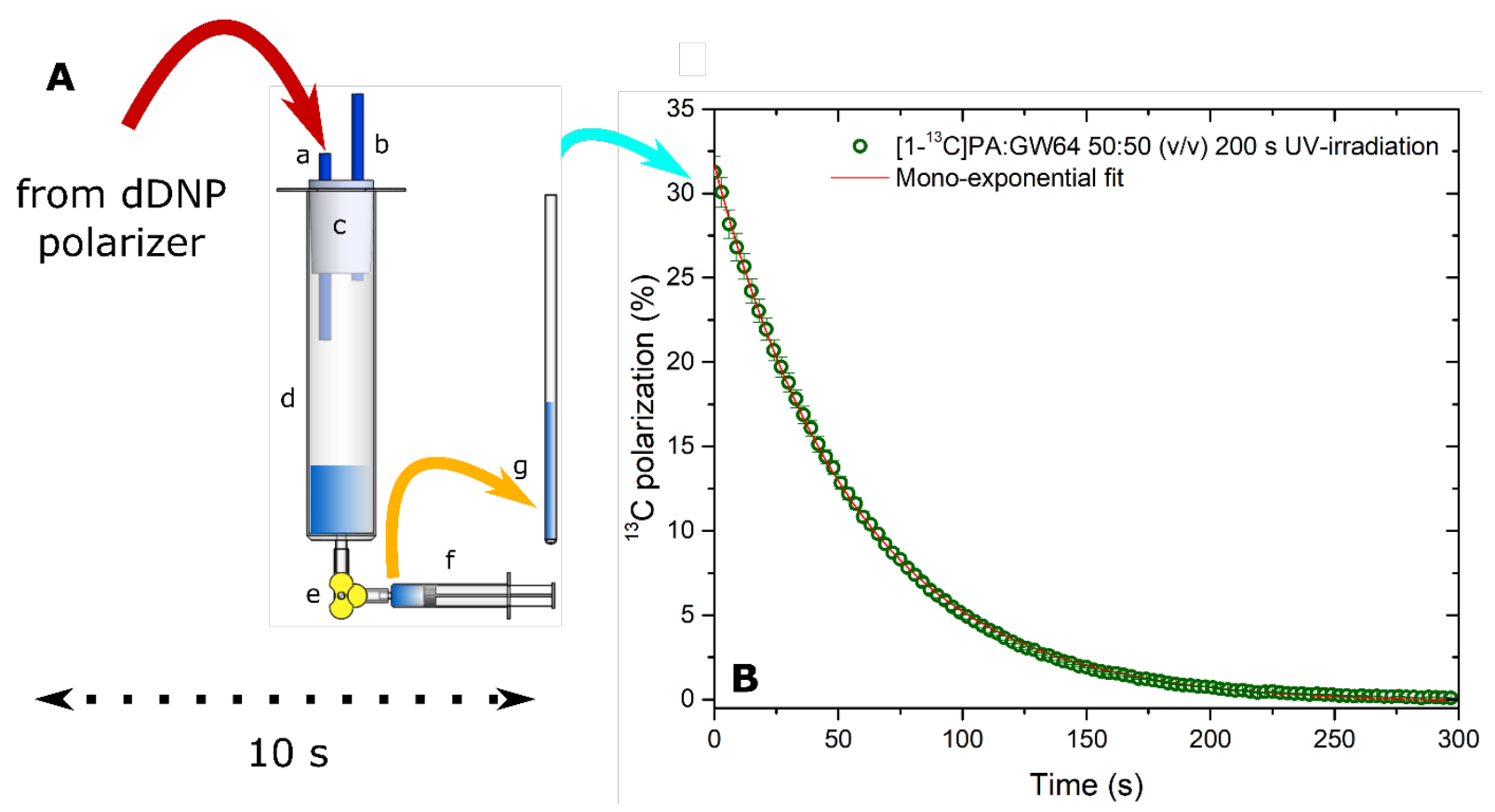

Figure 11A Manual receiving/injecting setup for the HP solution extracted from the dDNP polarizer composed by liquid inlet pipe (a); gas overpressure outlet pipe (b); pierced rubber plug (c); $50 \mathrm{~mL}$ syringe receiver (d); three-way valve (e); $2 \mathrm{~mL}$ syringe (f); $5 \mathrm{~mm}$ NMR tube (g). B Decay of the ${ }^{13} \mathrm{C}$ polarization inside the $400 \mathrm{MHz}$ spectrometer for the sample containing $\left[1-{ }^{13} \mathrm{C}\right] \mathrm{PA}: G W 64$ 50:50 (v/v) irradiated $200 \mathrm{~s}$; data were fitted with a mono-exponential curve.

\section{CONCLUSIONS}

In this work, we present a thorough investigation and optimization of the preparation of UVirradiated PA for dDNP, the most studied substrate for in vivo and in vitro ${ }^{13} \mathrm{C}$ hyperpolarized MR applications.

The principal aim was to reduce the long sample preparation time and increase the radical yield in order to make the DNP performance of the photo-induced non-persistent PA radical comparable 
to other currently used radical for dDNP. Another aim was to make available a robust protocol for producing/using these radicals for dDNP. With increased power and precursor tailored irradiation it was possible to double ( $>50 \mathrm{mM}$ ) the radical concentration ten times faster (in $5 \mathrm{~min}$ ) than previously reported. The higher radical concentration is essential for an efficient DNP process at high field. The latter together with microwave modulation tripled the maximum achievable polarization and reduced the buildup time constant to 25 min. A radical free PA solution polarized to $30 \%$ compares well with the standards currently employed for human experiments ( $34 \%{ }^{13} \mathrm{C}$ polarization on PA after Trityl radicals filtering and QC). ${ }^{39}$

\section{ASSOCIATED CONTENT}

- Dymax BlueWave 75 UV-lamp power calibration

- X-band ESR spectrometer calibration curve

- UV-induced radical stability

- LOD-ESR schematics and signal processing

- Radical generation time course

- Radical distribution profile

- Solid-state DNP buildups

This material is available free of charge via the Internet at http://pubs.acs.org.

\section{AUTHOR INFORMATION}




\section{Corresponding Author}

*Andrea Capozzi, e-mail: andcapo@elektro.dtu.dk

\section{Notes}

The authors declare no competing financial interests.

\section{ACKNOWLEDGMENTS}

We are grateful to Dr. Arnaud Comment, Dr. Christophe Roussel and Dr. Olivier Ouari for fruitful discussions. Our acknowledgement goes also to Mr. Jan Kilund from HYPERMAG for his constant help in solving technical and practical problems. This work was supported by the Danish National Research Foundation (DNRF124) and the H. C. Ørsted COFUND - Marie SkłodowskaCurie Postdoc Fellowship.

\section{REFERENCES}

1. Ardenkjaer-Larsen, J. H., On the Present and Future of Dissolution-Dnp. J Magn Reson 2016, 264, 3-12.

2. Bowen, S.; Hilty, C., Time-Resolved Dynamic Nuclear Polarization Enhanced Nmr Spectroscopy. Angew Chem Int Edit 2008, 47, 5235-5237.

3. Lee, Y.; Heo, G. S.; Zeng, H. F.; Wooley, K. L.; Hilty, C., Detection of Living Anionic Species in Polymerization Reactions Using Hyperpolarized Nmr. J Am Chem Soc 2013, 135, 46364639.

4. Lee, Y.; Zeng, H. F.; Ruedisser, S.; Gosser, A. D.; Hilty, C., Nuclear Magnetic Resonance of Hyperpolarized Fluorine for Characterization of Protein-Ligand Interactions. J Am Chem Soc 2012, 134, 17448-17451.

5. Lerche, M. H.; Jensen, P. R.; Karlsson, M.; Meier, S., Nmr Insights into the Inner Workings of Living Cells. Anal Chem 2015, 87, 119-132. 
6. Harris, T.; Eliyahu, G.; Frydman, L.; Degani, H., Kinetics of Hyperpolarized C-13(1)Pyruvate Transport and Metabolism in Living Human Breast Cancer Cells. P Natl Acad Sci USA 2009, 106, 18131-18136.

7. $\quad$ Golman, K.; in't Zandt, R.; Lerche, M.; Pehrson, R.; Ardenkjaer-Larsen, J. H., Metabolic Imaging by Hyperpolarized C-13 Magnetic Resonance Imaging for in Vivo Tumor Diagnosis. Cancer Res 2006, 66, 10855-10860.

8. Golman, K.; in't Zandt, R.; Thaning, M., Real-Time Metabolic Imaging. P Natl Acad Sci USA 2006, 103, 11270-11275.

9. Golman, K.; Petersson, J. S., Metabolic Imaging and Other Applications of Hyperpolarized C-13. Acad Radiol 2006, 13, 932-942.

10. Nelson, S. J., et al., Metabolic Imaging of Patients with Prostate Cancer Using Hyperpolarized [1-C-13]Pyruvate. Sci Transl Med 2013, 5, 198ra108 1-10.

11. Brindle, K. M., Imaging Metabolism with Hyperpolarized C-13-Labeled Cell Substrates. $J$ Am Chem Soc 2015, 137, 6418-6427.

12. Day, S. E.; Kettunen, M. I.; Gallagher, F. A.; Hu, D. E.; Lerche, M.; Wolber, J.; Golman, K.; Ardenkjaer-Larsen, J. H.; Brindle, K. M., Detecting Tumor Response to Treatment Using Hyperpolarized C-13 Magnetic Resonance Imaging and Spectroscopy. Nat Med 2007, 13, 13821387.

13. Kurhanewicz, J., et al., Analysis of Cancer Metabolism by Imaging Hyperpolarized Nuclei: Prospects for Translation to Clinical Research. Neoplasia 2011, 13, 81-97.

14. Overhauser, A. W., Polarization of Nuclei in Metals. Phys Rev 1953, 92, 411-415.

15. Carver, T. R.; Slichter, C. P., Polarization of Nuclear Spins in Metals. Phys Rev 1953, 92, 212-213.

16. Abragam, A.; Goldman, M., Principles of Dynamic Nuclear-Polarization. Rep Prog Phys 1978, 41, 395-467.

17. Ardenkjaer-Larsen, J. H.; Fridlund, B.; Gram, A.; Hansson, G.; Hansson, L.; Lerche, M. H.; Servin, R.; Thaning, M.; Golman, K., Increase in Signal-to-Noise Ratio of > 10,000 Times in Liquid-State Nmr. P Natl Acad Sci USA 2003, 100, 10158-10163.

18. Wolber, J., et al., Generating Highly Polarized Nuclear Spins in Solution Using Dynamic Nuclear Polarization. Nucl Instrum Meth A 2004, 526, 173-181. 
19. Ardenkjaer-Larsen, J. H.; Macholl, S.; Johannesson, H., Dynamic Nuclear Polarization with Trityls at 1.2 K. Appl Magn Reson 2008, 34, 509-522.

20. Lumata, L.; Merritt, M. E.; Kovacs, Z., Influence of Deuteration in the Glassing Matrix on C-13 Dynamic Nuclear Polarization. Phys Chem Chem Phys 2013, 15, 7032-7035.

21. Lumata, L.; Merritt, M. E.; Malloy, C. R.; Sherry, A. D.; Kovacs, Z., Impact of Gd3+ on Dnp of [1-C-13]Pyruvate Doped with Trityl Ox063, Bdpa, or 4-Oxo-Tempo. J Phys Chem A 2012, 116, 5129-5138.

22. Yoshihara, H. A. I.; Can, E.; Karlsson, M.; Lerche, M. H.; Schwitter, J.; Comment, A., High-Field Dissolution Dynamic Nuclear Polarization of [1-C-13]Pyruvic Acid. Phys Chem Chem Phys 2016, 18, 12409-12413.

23. Mieville, P., et al., Scavenging Free Radicals to Preserve Enhancement and Extend Relaxation Times in Nmr Using Dynamic Nuclear Polarization. Angew Chem Int Edit 2010, 49, 6182-6185.

24. Lipso, K. W.; Bowen, S.; Rybalko, O.; Ardenkjaer-Larsen, J. H., Large Dose Hyperpolarized Water with Dissolution-Dnp at High Magnetic Field. J Magn Reson 2017, 274, 65-72.

25. Kumada, T.; Noda, Y.; Hashimoto, T.; Koizumi, S., Dynamic Nuclear Polarization Study of Uv-Irradiated Butanol for Hyperpolarized Liquid Nmr. J Magn Reson 2009, 201, 115-120.

26. Capozzi, A.; Hyacinthe, J. N.; Cheng, T.; Eichhorn, T. R.; Boero, G.; Roussel, C.; van der Klink, J. J.; Comment, A., Photoinduced Nonpersistent Radicals as Polarizing Agents for X-Nuclei Dissolution Dynamic Nuclear Polarization. J Phys Chem C 2015, 119, 22632-22639.

27. Eichhorn, T. R.; Takado, Y.; Salameh, N.; Capozzi, A.; Cheng, T.; Hyacinthe, J. N.; Mishkovsky, M.; Roussel, C.; Comment, A., Hyperpolarization without Persistent Radicals for in Vivo Real-Time Metabolic Imaging. P Natl Acad Sci USA 2013, 110, 18064-18069.

28. Leermakers, P. A.; Vesley, G. F., Photolysis of Pyruvic Acid in Solution. J Org Chem $1963,28,1160-\&$.

29. Leermakers, P. A.; Vesley, G. F., Photochemistry of Alpha-Keto Acids and Alpha-Keto Esters .1. Photolysis of Pyruvic Acid and Benzoylformic Acid. J Am Chem Soc 1963, 85, 3776-\&.

30. Closs, G. L.; Miller, R. J., Photo-Reduction and Photodecarboxylation of Pyruvic-Acid Applications of Cidnp to Mechanistic Photochemistry. J Am Chem Soc 1978, 100, 3483-3494. 
31. Lewis, G. N.; Lipkin, D.; Magel, T. T., Reversible Photochemical Processes in Rigid Media. A Study of the Phosphorescent State. J Am Chem Soc 1941, 63, 3005-3018.

32. Guzman, M. I.; Colussi, A. J.; Hoffmann, M. R., Photogeneration of Distant Radical Pairs in Aqueous Pyruvic Acid Glasses. J Phys Chem A 2006, 110, 931-5.

33. Kendall, D. S.; Leermake.Pa, Photoreduction of Pyruvic Acid by Isopropyl Alcohol and TButyl Alcohol . A Kinetic Study. J Am Chem Soc 1966, 88, 2766-\&.

34. Guzman, M. I.; Colussi, A. J.; Hoffmann, M. R., Photoinduced Oligomerization of Aqueous Pyruvic Acid. J Phys Chem A 2006, 110, 3619-26.

35. Griffith, E. C.; Carpenter, B. K.; Shoemaker, R. K.; Vaida, V., Photochemistry of Aqueous Pyruvic Acid. Proc Natl Acad Sci U S A 2013, 110, 11714-9.

36. Capozzi, A.; Cheng, T.; Boero, G.; Roussel, C.; Comment, A., Thermal Annihilation of Photo-Induced Radicals Following Dynamic Nuclear Polarization to Produce Transportable Frozen Hyperpolarized 13c-Substrates. Nat Commun 2017, 8, 15757.

37. Hirsch, M. L.; Kalechofsky, N.; Belzer, A.; Rosay, M.; Kempf, J. G., Brute-Force Hyperpolarization for Nmr and Mri. J Am Chem Soc 2015, 137, 8428-8434.

38. Ardenkjaer-Larsen, J. H.; Leach, A. M.; Clarke, N.; Urbahn, J.; Anderson, D.; Skloss, T. W., Dynamic Nuclear Polarization Polarizer for Sterile Use Intent. Nmr Biomed 2011, 24, 927932.

39. Malinowski, R. M.; Lipso, K. W.; Lerche, M. H.; Ardenkaer-Larsen, J. H., Dissolution Dynamic Nuclear Polarization Capability Study with Fluid Path. J Magn Reson 2016, 272, 141146.

40. Granwehr, J.; Leggett, J.; Kockenberger, W., A Low-Cost Implementation of Epr Detection in a Dissolution Dnp Setup. J Magn Reson 2007, 187, 266-76.

41. Eugene, A. J.; Guzman, M. I., Reactivity of Ketyl and Acetyl Radicals from Direct Solar Actinic Photolysis of Aqueous Pyruvic Acid. J Phys Chem A 2017, 121, 2924-2935.

42. Eichhorn, T. R. Dynamic Nuclear Polarization with Paramagnetic Centers Created by Photo-Excitation. EPFL, 2014.

43. Fujisawa, T.; Monroe, B. M.; Hammond, G. S., Rates of Termination of Radicals in Solution .5. Ketyle Radicals Derived from Alpha-Keto Acids and Esters. J Am Chem Soc 1970, 92, 542-\&. 
44. Adeva, B., et al., Large Enhancement of Deuteron Polarization with Frequency Modulated Microwaves. Nucl Instrum Meth A 1996, 372, 339-343.

45. Kiselev, Y. F.; Niinikoski, T. O., Epr and Dynamic Nuclear Polarization with Frequency Modulation. In 12th International Symposium on High-energy Spin Physics : Spin '96,, Amsterdam, The Netherlands, 1996; pp 389-391.

46. Mims, W. B.; Nassau, K.; Mcgee, J. D., Spectral Diffusion in Electron Resonance Lines. Phys Rev 1961, 123, 2059-\&.

47. Bloembergen, N.; Shapiro, S.; Pershan, P. S.; Artman, J. O., Cross-Relaxation in Spin Systems. Phys Rev 1959, 114, 445-459.

48. Wenckebach, W. T., Spectral Diffusion and Dynamic Nuclear Polarization: Beyond the High Temperature Approximation. J Magn Reson 2017, 284, 104-114.

49. Bornet, A.; Milani, J.; Vuichoud, B.; Linde, A. J. P.; Bodenhausen, G.; Jannin, S., Microwave Frequency Modulation to Enhance Dissolution Dynamic Nuclear Polarization. Chem Phys Lett 2014, 602, 63-67.

50. Kenyon, W. E.; Day, P. I.; Stralay, C.; Willemsen, J. F., A Three-Part Study of Nmr Longitudinal Relaxation Properties of Water-Saturated Sandstones. Society of Petroleum Engineers 1988, 622-636.

51. Kurdzesau, F.; van den Brandt, B.; Comment, A.; Hautle, P.; Jannin, S.; van der Klink, J. J.; Konter, J. A., Dynamic Nuclear Polarization of Small Labelled Molecules in Frozen WaterAlcohol Solutions. J Phys D Appl Phys 2008, 41, 155506 1-10.

52. Cheng, T.; Capozzi, A.; Takado, Y.; Balzan, R.; Comment, A., Over 35\% Liquid-State 13c Polarization Obtained Via Dissolution Dynamic Nuclear Polarization at $7 \mathrm{~T}$ and $1 \mathrm{~K}$ Using Ubiquitous Nitroxyl Radicals. Phys Chem Chem Phys 2013, 15, 20819-22.

\section{TOC GRAPHICS}




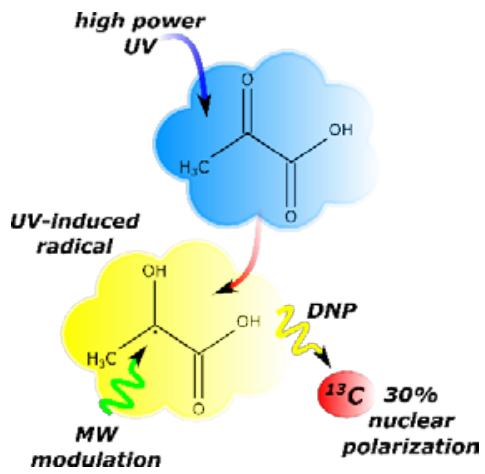

